\title{
In-patient management of men with schizophrenia who commit serious sexual offences
}

\author{
Alan D. Smith
}

\begin{abstract}
Aims and method A survey of the management of all 84 restricted hospital order in-patients with schizophrenia, resident in any hospital in England and Wales, during May 1997, with an index conviction for a contact sex offence against a woman, was carried out. Results Treatment concentrated upon pharmacological therapy for psychiatric symptoms. Only a small minority of patients received specific psychological therapy to address sexual offending issues, despite responsible medical officers reporting that over fourfifths of patients continued to have marked social or sexual difficulties relating to women, and that nonpsychotic factors appeared to make a significant contribution to index sexual offending in about threequarters of cases.

Clinical implications Many in-patients with schizophrenia who have committed sexual offences may not be recelving optimal management and more research is required to elucidate their treatment needs.
\end{abstract}

Strategies to address the problems of nonmentally ill sex offenders may include cognitivebehavioural, psychodynamic and physical therapies, and much research continues to be associated with the formulation and evaluation of treatment programmes for such offenders (Barker \& Morgan, 1993; Beckett et al, 1994).

In contrast, there is an absence of systematic knowledge concerning the types of treatment given to men with major mental illness, such as schizophrenia, who have committed serious sexual offences. It has been suggested that the management of sex offenders with schizophrenia typically involves treatment aimed at resolving psychotic symptoms with little attention to addressing factors such as deviant sexual interest and distorted cognitions, despite preliminary evidence indicating that such offenders, similar to their sex offending peers who are not mentally ill, may have high levels of sexual obsession, sexual dysfunction, faulty sexual knowledge and beliefs (Sahota \& Chesterman. 1998).

This paper reports on the management of a national sample of hospitalised men with schizo- phrenia who have been convicted of a contact sex offence against a woman, and who have been considered by the court to pose a special risk to the public such that a restriction order has been imposed.

\section{The study}

This treatment survey formed part of a larger investigation into the relationship between schizophrenia and sex offending. The Mental Health Unit (MHU) of the Home Office has responsibility for all mentally disordered offenders detained under the Mental Health Act 1983 and subject to restrictions on discharge. A search of MHU files was made for all male in-patients resident in any hospital in England and Wales during May 1997 with an ICD-10 (World Health Organization, 1992) diagnosis of schizophrenia, following conviction for an index sex offence of rape, attempted rape or indecent assault of a female 16 years old and above. The MHU files are large dossiers containing detailed information about previous offending, psychiatric and social history, official correspondence including minutes of clinical case conferences, treatment plans, mental health review tribunal reports and outcomes, and progress reports submitted at regular intervals by the responsible medical officer (RMO).

A check-list, specially developed for the study, was applied to the files of all patients by me. Most of the information collected was factual or clinical opinion as reported by the RMO and multi-disciplinary team. As a check on the reliability of coding, the files of 10 randomly selected files were re-rated by the researcher at the end of the study without reference to the first rating. There were no significant differences between the first and second coding.

\section{Findings}

There were 84 restricted hospital order inpatients with schizophrenia, resident in any 
hospital in England and Wales, during May 1997, following conviction for a contact sex offence against a woman.

\section{Location and general features}

Thirty-one (37\%) patients were being treated under conditions of high security in one of three special hospitals (Broadmoor in Berkshire, Rampton in Nottinghamshire and Ashworth in Merseyside), $45(53 \%)$ in one of 25 regional (medium) secure units (RSUs) distributed throughout England and Wales and four (5\%) each in locked and open wards of general psychiatric hospitals. During this period of continuous in-patient treatment, just under half (37 (44\%)) of the patients had been transferred. on at least one occasion, to a hospital with a different level of security, such that $35(42 \%)$ patients had spent time in a special hospital, 60 $(71 \%)$ in a RSU, $12(14 \%)$ in a locked ward and 11 $(13 \%)$ in an open ward. A total of $23(27 \%)$ patients had been transferred to a hospital with a higher level of security and in 17 of these cases. sexually inappropriate behaviour or concern about the risk of such behaviour was a significant factor leading to transfer.

At the time of data collection the median length of hospital stay was 50 months (mean 57, range 4-165) with $87 \%$ men having been in-patients for at least one year. The duration of in-patient stay for those men who had spent time in a special hospital (median 80 months, mean 78 months, range 6-165 months) was significantly longer than that for patients who had not been treated under high security (median 38, mean 41 , range 4-126; Mann-Whitney $U$-test, $z=-3.6$, $P=0.0003$ ).

\section{Assessment}

With the exception of two cases, all men underwent, in addition to standard psychiatric assessments, more detailed evaluations of previous sexual relationships, interests and past sexual offending. For $14(17 \%)$ patients, assessments were supplemented by the use of specific psychosexual rating scales. Only four patients, all in special hospitals, were assessed using penile plethysmography, a psychophysiological measure of sexual arousal.

In $69(82 \%)$ cases the RMO and multi-disciplinary team considered that the patient had continuing social and sexual difficulties relating to women and $28(33 \%)$ were viewed as having deficient sexual knowledge. The RMO reported that in $22(26 \%)$ patients the index sex offence appeared to be entirely or principally attributable to the effects of psychotic mental illness (delusions/hallucinations/disinhibition), while in the majority $(62(74 \%))$ of patients, either a combination of psychotic and non-psychotic features (e.g. anger, deviant sexual interest) or non-psychotic features alone were thought to be relevant.

\section{Treatment}

With regard to pharmacological therapy, all patients received antipsychotic medication (oral or depot) and 12 had or were being treated with clozapine. Only one patient was prescribed antilibidinal medication in the form of cyproterone acetate.

All patients except one, received some form of non-specific individual talking treatment of varying intensity, typically supportive psychotherapy from a key nurse, and most patients attended some form of off-ward activity (e.g. occupational therapy). Only 10 (12\%) men underwent individual cognitive-behavioural treatment to specifically address sexual offending and social dysfunction, and four of these received treatment packages focusing upon sex education and development of social skills. One patient received individual psychodynamic therapy. Nine (11\%) patients had some form of group therapy but for only six patients was this specifically linked to sexual offending issues. Specific group therapy occurred in special hospitals only, while individual cognitivebehavioural work was as likely to occur in RSUs as special hospitals (none in locked or open wards of general psychiatric hospitals).

\section{Comment}

The majority of the sex offender patients studied were being treated under conditions of at least medium security for considerable periods of time, especially those in special hospital, emphasising the importance of ensuring that the most appropriate forms of treatment are delivered during these relatively expensive, by general psychiatric standards, in-patient stays.

The emphasis upon pharmacological treatment of schizophrenia in this forensic population of seriously ill men is unsurprising. However, the small proportion of men receiving specific psychological therapy to address sexual offending issues, either at an individual or group level, is notable, especially as the RMO and clinical teams reported continuing social or sexual deficits in about four-fifths of patients, and non-psychotic factors to have contributed significantly to sexual offending in about threequarters of cases. A sizeable minority continued to exhibit problematic sexual behaviour while in hospital.

Although there is continuing debate about the efficacy of different methods of treating sex offenders (Marshall, 1993; Quinsey et al, 1994). 
clinicians treating individuals without mental illnesses have increasingly adopted a cognitivebehavioural approach, employing a package of interventions dealing with sex education, social skills, interpersonal functioning, victim empathy and relapse prevention (Marshall \& Barbaree, 1990). Why was such treatment not being given more widely to these sex offenders with schizophrenia? In some cases, the RMO reported that severe ongoing psychotic symptoms precluded the patient engaging in any structured psychological therapy on a sustained basis. However, in many others, the treatment plans gave little, if any, consideration to specific psychological treatment to address sexual offending. In some cases the RMO reported that individual or group cognitive-behavioural work was indicated, but not given, due to lack of key personnel or placement on long waiting lists of psychology departments. The lack of specific psychological input, for example, to address social skills difficulties is even more surprising when it is considered that deficits thought to be associated with sex offending may be complicated by the detrimental effects of schizophrenia itself upon social functioning.

In general, patients underwent relatively detailed assessments of their sexuality. The MHU staff, through their correspondence with the RMO, increasingly have a proactive role in ensuring that each patient undergoes a sufficiently thorough assessment. Areas which tended not to receive systematic attention included specific enquiry into the full range of paraphilic behaviours or interests, evaluation of current sexual fantasies and intensity of sexual drive. More widespread use of appropriate rating scales may lead to capture of such information and may be more acceptable to some patients who prefer neutral methods of inquiry rather than face-to-face interviews with clinicians (Sahota \& Chesterman, 1998).

As a focus of research, sex offenders with schizophrenia have been very neglected. Much more work is required to elucidate the needs of such patients and to ensure that they receive optimal treatment.

\section{References}

BARKER, M. \& MORGAN, R. (1993) Sex Offenders: a Framework for the Evaluation of Community-Based Treatment. A Report for the Home Office. London: Home Office.

BECKETT, R., BEECH, A., FISHER, D., et al (1994) Community Based Treatment for Sex Offenders: An Eualuation of Seven Treatment Programs. Report for the Home Office by the STEP team. London: Home Office.

MARSHALL, W. L. (1993) The treatment of sex offenders. What does the outcome data tell us? Joumal of Interpersonal Violence, 8, 524-530.

- \& BARBAREE, H. E. (1990) An integrated theory of the etiology of sexual offending. In Handbook of Sexual Assault: Issues. Theories, and Treatment of the Offender (eds W. L. Marshall. D. R. Laws \& H. E. Barbaree), pp. 257-275. New York: Plenum.

QUINSEY, V. L., HARRIS, G. RICE, M. I. et al (1993) Assessing treatment efficacy in outcome studies of sex offenders. Joumal of Interpersonal Violence, 8, 512-523.

SAHOTA, K. \& ChEsterman, P. (1998) Mentally ill sex offenders in a reglonal secure unit. II: cognitions, perceptions and fantasies. Journal of Forensic Psychiatry, 9, 161-172.

WORLD HEALTH ORGANIZATION (1992) The Tenth Revision of the International Classification of Diseases and Related Health Problems (ICD-10). Geneva: WHO.

Alan D. Smith, Lecturer in Forensic Psychiatry, Department of Forensic Psychiatry. Institute of Psychiatry. De Crespigny Park, London SE5 8AF 